\title{
ON MEROMORPHIC FUNCTIONS COMMUTING WITH ELEMENTS OF A FUNCTION GROUP ${ }^{1}$
}

\author{
JOHN RODERICK SMART
}

\begin{abstract}
The problem of whether there always exist meromorphic functions commuting with all substitutions of a function group is solved in the affirmative.
\end{abstract}

1. Introduction. Maurice Heins studied [4], [5] mapping properties of meromorphic functions $F$ in the upper half plane, Im $\tau>0$, which commute with all substitutions of the modular group $M$. Thus,

$$
F\left(\frac{a \tau+b}{c \tau+d}\right)=F(V \tau)=V F(\tau)=\frac{a F(\tau)+b}{c F(\tau)+d}
$$

for each $V=\left(\begin{array}{ll}a & b \\ c & d\end{array}\right)$, with $a, b, c, d$ rational integers and $a d-b c=1$. Brady [1], a student of Heins, parametrized all solutions of the above functional equations in terms of a complex parameter and an unrestricted modular function $A$, that is, a meromorphic function in $\operatorname{Im} \tau>0$ satisfying $A(V \tau)=$ $A(\tau)$ for each $V$ in $M$. Brady in the same paper generalized this result to the following extent. Let $\Gamma$ be a function group with domain of discontinuity $\mathscr{D}$ and suppose $F_{1}, F_{2}$ and $F_{3}$ are three distinct functions which commute with the elements of $\Gamma$. Then for any $F \neq F_{1}$ which also commutes with the elements of $\Gamma$, the cross ratio $A(\tau)=\left[\left(F_{1}-F_{2}\right)\left(F_{3}-F\right) /\left(F_{3}-F_{2}\right)\left(F_{1}-F\right)\right]$ is an automorphic function for $\Gamma$, and so

$$
F=\frac{F_{2}\left(F_{1}-F_{3}\right)+(A-1)\left(F_{1}-F_{3}\right)}{\left(F_{1}-F_{3}\right)+(A-1)\left(F_{2}-F_{3}\right)} .
$$

Brady's result is only quoted for Fuchsian groups but the proof is valid in the more general situation. Outside the case $\Gamma=M$, he had no way of constructing functions $F$ commuting with the transformations in $\Gamma$. In $\$ 2$ we show how to construct such functions from automorphic forms and how to get three linearly independent such functions. In $\S 3$ we treat the case of

Received by the editors March 16, 1971.

AMS 1970 subject classifications. Primary 30A58; Secondary 30A20, 10D05, 10D15, $33 \mathrm{~A} 25$.

Key words and phrases. Automorphic form, Fuchsian group, Kleinen group, Weierstrass zeta function, modular group, Dedekind eta function.

1 Work supported by National Science Foundation grant GP-20219. 
the modular group $M$ specially and show the motivation for the functions defined in $\$ 2$.

We now introduce some definitions and notation. The general reference for this material is either Lehner [8] or Ford [2]. Let $\Gamma$ be an infinite group of two by two matrices with complex entries containing $-I, I=\left(\begin{array}{l}1 \\ 0 \\ 0\end{array}\right)$, for which the associated group of linear fractional transformations $\Gamma=$ $\left\{V \tau=(a \tau+b) /(c \tau+d): V=\left(\begin{array}{ll}a & b \\ c & d\end{array}\right) \in \Gamma\right\}$ is a function group. That is, there is a domain $\mathscr{D}$ of ordinary points of $\Gamma$ carried into itself by the elements of $\Gamma$, and the boundary of $\mathscr{D}$ consists of limit points of $\Gamma$. Such groups comprise the so-called Fuchsian and Kleinen groups. Two points $\tau_{1}, \tau_{2}$ of $\mathscr{D}$ are equivalent if there is a $V$ in $\Gamma$ such that $V \tau_{1}=\tau_{2}$. Let $\Gamma \tau$, the orbit of $\tau$, denote the set of images of $\tau$ by elements of $\Gamma$.

Let $r$ be a real number; then a function $v: \Gamma \rightarrow\{\tau:|\tau|=1\}$ is called a multiplier system for $\Gamma$ and $-r$ if a certain consistency condition [8, p. 267] is satisfied. If $r$ is integral, then $v$ is a character on $\Gamma$. A function $f$ meromorphic on $\mathscr{D}$ which satisfies

$$
f(V \tau)=v(V)(c \tau+d)^{r} f(\tau), \quad \tau \in \mathscr{D},
$$

for each $V=\left(\begin{array}{ll}a & b \\ c & d\end{array}\right) \in F$, is called an unrestricted automorphic form of weight $-r$ for $\Gamma$ and $-r$. Here $(c \tau+d)^{r}=\exp \{r \log (c \tau+d)\}$ with $-\pi \leqq \arg (c \tau+d)<\pi$. If $f$ has a finite number of poles in a fundamental region $R(\subset \mathscr{D})$ then $f$ is called an automorphic form. If $r=0$ then $f$ is called an automorphic function. The set of these automorphic forms form a complex vector space denoted by $\{\Gamma,-r, v\}$.

On certain function groups $\Gamma$ one can construct automorphic forms by a method introduced by Poincaré [8, v. 2]. Let $H(z)$ be a rational function whose poles are not at limit points of $\Gamma$ nor in the orbit of $\infty$. If $m \geqq 2$ the series

$$
\theta(z ; H)=\sum H(V z) /(c z+d)^{2 m},
$$

where summation is over all $V=\left(\begin{array}{ll}a & b \\ c & d\end{array}\right)$ in $\Gamma$ ( $\infty$ is not fixed by any element of $\Gamma$ ), converges uniformly and absolutely on compact subsets of $\mathscr{D}$ not containing any points equivalent to the poles of $H$ or to $\infty . \theta(z ; H) \in$ $\{\Gamma,-2 m, 1\}$. By use of the $A$-transform [5, v. 3] one can extend the construction of forms to the remaining function groups.

Now we turn to the Weierstrass zeta function. Let $\omega_{1}, \omega_{2}$ be complex numbers such that $\operatorname{Im} \omega_{2} / \omega_{1}>0$. Then $\zeta\left(\tau ; \omega_{1}, \omega_{2}\right)$ is defined by

$$
\zeta\left(\tau ; \omega_{1}, \omega_{2}\right)=1 / \tau^{2}+\sum_{\omega \neq 0}\left\{1 /\left(\tau-(\omega)+1 / \omega+\tau^{2} / \omega^{2}\right\},\right.
$$

where summation is over all periods $\omega=m()_{1}+n \omega_{2}$ with $m, n$ integers not 
both 0 . Then for all $\tau$,

$$
\zeta\left(\tau+\omega_{1}\right)=\zeta(\tau)+\eta_{1}, \quad \zeta\left(\tau+\omega_{2}\right)=\zeta(\tau)+\eta_{2},
$$

where $\eta_{1}=\eta_{1}\left(\omega_{1}, \omega_{2}\right)$ and $\eta_{2}=\eta_{2}\left(\omega_{1}, \omega_{2}\right)$ are the pseudoperiods. They satisfy Legendre's relation $[9$, p. 376]

$$
\omega_{2} \eta_{1}-i_{1} \eta_{2}=2 \pi i \text {. }
$$

It follows from Heins study [5] of functions of type (1.1) that an ordered pair of complex numbers $\left(\eta_{1}, \eta_{2}\right) \neq(0,0)$ is the pair of pseudoperiods for a suitable Weierstrass zeta function.

I would like to thank the referee for pointing out an error in the earlier version and the reference to Hurwitz's work.

2. In this section we show how to derive, from automorphic forms, meromorphic functions $F$ satisfying (1.1) for the transformations in a function group $\Gamma$. We further show how to construct three linearly independent such functions. Now we prove the main theorem.

THEOREM 1. Let $\Gamma$ be a function group with domain of discontinuity $\mathscr{D}$, $v$ a multiplier system for $\Gamma$ and $-r$, and $f \in\{\Gamma,-r, v\}$. Then

$$
F(\tau)=r f(\tau) / f^{\prime}(\tau)+\tau
$$

commutes with all substitutions of $\Gamma$.

ProOF. Let $V=\left(\begin{array}{ll}a & b \\ c & d\end{array}\right) \in \Gamma$ then $f(V \tau)=v(V)(c \tau+d)^{r} f(\tau)$. Differentiating this relation with respect to $\tau$ and noting that $d V \tau / d \tau=1 /(c \tau+d)^{2}$, we obtain

$$
f^{\prime}(V \tau)=v(V)\left[r(c \tau+d)^{r+1} c f(\tau)+(c \tau+d)^{r+2} f^{\prime}(\tau)\right]
$$

Thus

$$
\begin{aligned}
F(V \tau) & =\frac{r f(\tau)}{(c \tau+d)\left[c r f(\tau)+(c \tau+d) f^{\prime}(\tau)\right]}+\frac{a \tau+b}{c \tau+d} \\
& =\frac{[1+c(a \tau+b)] r f+(a \tau+b)(c \tau+d) f^{\prime}}{(c \tau+d)\left[c r f+(c \tau+d) f^{\prime}\right]} \\
& =\frac{[a d-b c+c(a \tau+b)] r f+(a \tau+b)(c \tau+d) f^{\prime}}{(c \tau+d)\left[c r f+(c \tau+d) f^{\prime}\right]} \\
& =\frac{a\left(r f+\tau f^{\prime}\right)+b f^{\prime}}{c\left(r f+\tau f^{\prime}\right)+d f^{\prime}}=V(F \tau) .
\end{aligned}
$$

LEMMA 1. Let $\Gamma$ be a function group, then there are points $\tau_{1}, \tau_{2}$ and $\tau_{3} \in \mathscr{D}$, which are pairwise inequivalent; and forms $f_{1}$ and $f_{2} \in\{\Gamma,-4,1\}$ so that $f_{1}$ has simple poles at $\tau_{1}$ and $\tau_{2}$ and is regular at $\tau_{3}$, and $f_{2}$ has simple poles at $\tau_{1}$ and $\tau_{3}$ and is regular at $\tau_{2}$. 
Proof. Suppose first $\Gamma$ is a function group for which $\infty$ is an ordinary point and $\infty$ is not fixed by any element of $\Gamma$. Let $\tau_{1}, \tau_{2}, \tau_{3}$ be chosen so that they are inequivalent and none belongs to the orbit of $\infty$. Let $H_{1}(\tau)=$ $1 /\left(\tau-\tau_{1}\right)\left(\tau-\tau_{2}\right)$ and $H_{2}(\tau)=1 /\left(\tau-\tau_{1}\right)\left(\tau-\tau_{3}\right)$. Then $\theta\left(\tau ; H_{i}\right)$ defined in (1.4) with $m=2$ belongs to $\{\Gamma,-4,1\}$. Moreover, one can see by the proof of the Theorems V.2.D and V.2.E of Lehner [8, pp. 160-161] that $\theta\left(\tau ; H_{i}\right)$ has simple poles at the required points. Moreover, by the choice of the $\tau_{i}$, $\theta_{1}$ is regular at $\tau_{3}$, and $\theta_{2}$ is regular at $\tau_{2}$. Now if $\Gamma$ does not have the required properties at $\infty$ we consider $A \Gamma A^{-1}$ for a real matrix $A$ of determinant 1 . The region of discontinuity of $A \Gamma A^{-1}$ is $A \mathscr{D}$ and the fixed points $\left\{\zeta_{i}\right\}$ of the transformations of $A \Gamma A^{-1}$ are $\left\{A \tau_{i}\right\}$ where $\left\{\tau_{i}\right\}$ are the fixed points of the transformations of $\Gamma$. Thus we can pick an $A$ so that $A \Gamma A^{-1}$ has the required properties at $\propto$. Then solving as above we obtain points $\zeta_{1}, \zeta_{2}, \zeta_{3} \in \mathscr{D}$ and functions $g_{1}, g_{2}$ with the properties of the theorem. Now consider $f_{i}=g_{i} \mid A^{-1}=g_{i}(A \tau)(d A \tau / d \tau)^{m} ; f_{i}$ is an automorphic form on $A^{-1}\left(A \Gamma A^{-1}\right) A=\Gamma\left[8\right.$, p. 164]. Clearly, $f_{1}$ has poles at $\tau_{1}=A^{-1} \zeta_{1}$ and $\tau_{2}=A^{-1} \zeta_{2}$ and is regular at $A^{-1} \tau_{3} . f_{2}$ has similar properties.

THEOREM 2. Let $\Gamma$ be a function group then there exist three linearly independent functions which commute with all substitutions of $\Gamma$.

Proof. Pick $0 \neq \tau_{1}, \tau_{2}, \tau_{3}$, and $f_{1}$ and $f_{2}$ as in Lemma 1. By Theorem 1, $F_{i}=4\left(f_{i} \mid f_{i}^{\prime}\right)+\tau, i=1,2$, commutes with all substitutions of $\Gamma$. Let $F_{3}(\tau)=\tau$, be the identity function. Suppose

$$
a F_{1}+b F_{2}+c F_{3}=0 .
$$

We see that $f_{i} / f_{i}^{\prime}$ has a zero at $\tau=\tau_{1}$, so setting $\tau=\tau_{1}$ in the above equation we have $a+b+c=0$. Thus the above equation reduces to

$$
a f_{1} / f_{1}^{\prime}+b f_{: 2} / f_{2}^{\prime}=0 .
$$

However, $f_{1}^{\prime} / f_{1}$ and $f_{2}^{\prime} / f_{2}$ are linearly independent since they have poles at different places, thus their reciprocals are linearly independent. So $a=b=$ $0=c$.

REMARK. One can now apply the parametrization theorem of Brady quoted in the introduction to parametrize as in (1.2) all $F$ 's commuting with elements of $\Gamma$ in terms of $F_{1}, F_{2}, F_{3}$ and unrestricted automorphic functions $A$.

3. In this section we give the motivation for the definition of $F$ given in (2.1). Let $M$ denote the (homogeneous) modular group, the $2 \times 2$ matrices with rational integer entries and determinant $1 . \bar{M}$ denotes the corresponding group of linear fractional transformations. 
THEOREM 3. Let $f \in\{M,-r, v\}$ and define

$$
g_{1}(\tau)=f^{\prime}(\tau) / f(\tau), \quad g_{2}(\tau)=g_{1}(-1 / \tau) / \tau
$$

then $F(\tau)=g_{2}(\tau) / g_{1}(\tau)$ commutes with the elements of $\bar{M}$.

Proof. It is an easy calculation to show that $F(\tau)=r f(\tau) / f^{\prime}(\tau)+\tau$, and thus this is a special case of Theorem 1. However, we prefer to give an alternate proof. $M$ is known to be generated by $U=\left(\begin{array}{ll}1 & 1 \\ 0 & 1\end{array}\right)$ and $T=\left(\begin{array}{cc}0 & -1 \\ 1\end{array}\right)$; thus it suffices to prove $F(U \tau)=F(\tau)+1$ and $F(T \tau)=-1 / F(\tau)$. First, from the definitions, $F(-1 / \tau)=-\tau g_{1}(\tau) / \tau g_{2}(\tau)=-1 / F(\tau)$. We also easily find that

or

$$
g_{1}(-1 / \tau)=r \tau+\tau^{2} g_{1}(\tau)
$$

$$
\tau g_{1}(\tau)-g_{2}(\tau)=-r
$$

If Legendre's relation (1.7) were written in inhomogeneous notation it would have the above form, so we could call (3.2) a generalization of Legendre's formula. Now $g_{1}(\tau+1)=g_{1}(\tau)$ so that $g_{2}(\tau+1)=(\tau+1) g_{1}(\tau+1)+r=$ $\tau g_{1}(\tau)+g_{1}(\tau)+r=g_{1}(\tau)+g_{2}(\tau)$. It follows that $F(\tau+1)=F(\tau)+1$.

We now turn to the pseudoperiods of the Weierstrass zeta function and the investigation which led to the definitions (2.1) and (3.1). Our method will be similar to one used by Hurwitz [6], [7] to obtain the transformation properties of the discriminant $\Delta(\tau)$ in the theory of elliptic modular forms. We shall consider $\eta_{1}=\zeta\left(z+\omega_{1}\right)-\zeta(z)$. In order to obtain an expression for $\eta_{1}$ we sum the absolutely convergent series (1.5) for $\zeta$ over all $\omega=m \omega_{1}+$ $n \omega_{2}$ first over all $m \in Z$ and then over $n \in Z$. In the expression for $\eta_{1}$ obtained in this way we get a series which is telescopic and obtain after simplification

$$
\eta_{1}=\sum_{n}^{\prime} \sum_{m} \frac{\omega_{1}}{\left(m \omega_{1}+n \omega_{2}\right)^{2}}+\frac{1}{\omega_{1}} \frac{\pi^{2}}{3}
$$

where the dash denotes that the term $n=0$ is missing and the conditionally convergent sums are understood in the sense of Cauchy principal values. Similarly we obtain

$$
\eta_{2}=\sum_{m}^{\prime} \sum_{n} \frac{\omega_{2}}{\left(m \omega_{1}+n \omega_{2}\right)^{2}}+\frac{1}{\omega_{2}} \frac{\pi^{2}}{3} .
$$

If we now suppose the periods are normalized so that $\omega_{2}=\tau, \omega_{1}=1$ and $\operatorname{Im} \tau>0$, we obtain

$$
\begin{aligned}
& \eta_{1}(\tau)=\sum_{n}^{\prime} \sum_{m}^{\prime} \frac{1}{(m+n \tau)^{2}}+\frac{\pi^{2}}{3}\left(1+\frac{1}{\tau^{2}}\right), \\
& \eta_{2}(\tau)=\tau \sum_{m}^{\prime} \sum_{n}^{\prime} \frac{1}{(m+n \tau)^{2}}+\frac{\pi^{2}}{3}\left(\tau+\frac{1}{\tau}\right),
\end{aligned}
$$


where $\eta_{1}(\tau)=\eta_{1}(1, \tau), \eta_{2}(\tau)=\eta_{2}(1, \tau)$, and the dash on the summation sign means that the summation variable omits the value 0 . It is interesting that Legendre's relation (1.7) implies the following difference in value of conditionally convergent series:

$$
\sum_{n}^{\prime} \sum_{m}^{\prime}(m+n \tau)^{-2}-\sum_{m}^{\prime} \sum_{n}^{\prime}(m+n \tau)^{-2}=2 \pi i / \tau .
$$

A slightly more general result was derived by Hurwitz [6, p. 349]. It follows from (3.5) and (3.6) that

$$
\eta_{1}(-1 / \tau)=\tau \eta_{2}(\tau)
$$

This is the motivation for the definition of $g_{2}$ in (3.1). There is a known connection [3, p. 313] between $\eta_{1}$ and the Dedekind $\eta$-function. Dedekind's $\eta$ is defined by

$$
\eta(\tau)=e^{\pi i \tau / 12} \prod_{n=1}^{\infty}\left(1-e^{2 \pi i n \tau}\right), \quad \operatorname{Im} \tau>0 .
$$

Fricke proved that

$$
\eta_{1}(\tau)=-4 \pi i\left(\eta^{\prime}(\tau) / \eta(\tau)\right)=-4 \pi i(\log \eta(\tau))^{\prime} .
$$

This motivates the definition (3.1). Thus the choice of $f(\tau)=\eta(\tau)$ for Theorem 3 leads to the function considered by Heins [4].

\section{REFERENCES}

1. M. Brady, Meromorphic solutions of a system of functional equations involving the modular group, Proc. Amer. Math. Soc. 30 (1971), 271-277.

2. Lester R. Ford, Automorphic functions, McGraw-Hill, New York, 1929.

3. R. Fricke, Die elliptishe Funktionen und ihre Anwendungen. I, Teubner, Leipzig, 1916.

4. M. Heins, On the pseudoperiods of the Weierstrass zeta functions, SIAM J. Numer. Anal. 3 (1966), 266-268. MR 34 \#4548.

5. - On the pseudo-periods of the Weierstrass zeta functions. II, Nagoya Math. J. 30 (1967), 113-119. MR 41 \#7098.

6. A. Hurwitz. Grundlagen einer independenten Theorie der elliptischen Modulfunktionen und die Theorie der Multiplikator-Gleichungen erster Stufe, Math. Ann. 18 (1881), 528-592; Werke. I, Basel, 1932, pp. 1-66.

7. - Über die Theorie der elliptischen Modulfunktionen, Math. Ann. 58 (1904), 343-360; Werke. I, Base1, 1932, pp. 577-595.

8. J. Lehner, Discontinuous groups and automorphic functions, Math. Surveys, no. 8, Amer. Math. Soc., Providence, R. I., 1964. MR 29 \#1332.

9. S. Saks and A. Zygmund, Analytic functions, 2nd ed., PWN, Warsaw, 1965. MR 31 \#4889.

Department of Mathematics, University of Wisconsin, Madison, Wisconsin 53706 